\title{
Radon Numbers Grow Linearly
}

\author{
Dömötör Pálvölgyi ${ }^{1}$
}

Received: 6 July 2020 / Revised: 11 July 2021 / Accepted: 11 July 2021 /

Published online: 27 August 2021

(c) The Author(s) 2021

\begin{abstract}
Define the $k$-th Radon number $r_{k}$ of a convexity space as the smallest number (if it exists) for which any set of $r_{k}$ points can be partitioned into $k$ parts whose convex hulls intersect. Combining the recent abstract fractional Helly theorem of Holmsen and Lee with earlier methods of Bukh, we prove that $r_{k}$ grows linearly, i.e., $r_{k} \leq c\left(r_{2}\right) \cdot k$.
\end{abstract}

Keywords Convexity space $\cdot$ Radon numbers $\cdot$ Helly theorem $\cdot$ Tverberg theorem

\section{Introduction}

Define a convexity space as a pair $(X, \mathcal{C})$, where $X$ is any set of points and $\mathcal{C}$, the collection of convex sets, is any family over $X$ that contains $\varnothing, X$, and is closed under (arbitrary) intersection and under (arbitrary) union of nested sets. The convex hull, conv $S$, of some point set $S \subset X$ is defined as the intersection of all convex sets containing $S$, i.e., conv $S=\bigcap\{C \in \mathcal{C} \mid S \subset C\}$; since $\mathcal{C}$ is closed under intersection, conv $S$ is the minimal convex set containing $C$. This generalization of the convex sets of $\mathbb{R}^{d}$ includes several examples; for an overview, see the book by van de Vel [29] or, for a more recent work, [22]. It is a natural question what properties of convex sets of $\mathbb{R}^{d}$ are preserved, or what the relationships are among them for general convexity spaces. A much investigated parameter is the Radon number $r_{k}$ (sometimes also called partition number or Tverberg number), which is defined as the smallest number (if it exists) for which any set of $r_{k}$ points can be partitioned into $k$ parts whose convex hulls intersect in a common point. For $k=2$, we simply write $r=r_{2}$.

Editor in Charge: János Pach

Research supported by the Lendület program of the Hungarian Academy of Sciences (MTA), under Grant Number LP2017-19/2017.

Dömötör Pálvölgyi

domotorp@gmail.com

1 MTA-ELTE Lendület Combinatorial Geometry Research Group, Institute of Mathematics, Eötvös Loránd University (ELTE), Budapest, Hungary 
In case of the convex sets of $\mathbb{R}^{d}$, it was shown by Radon [26] that $r=d+2$ and by Tverberg [28] that $r_{k}=(d+1)(k-1)+1$. Calder [8] and Eckhoff [12] raised the question whether $r_{k} \leq(r-1)(k-1)+1$ also holds for general convexity spaces (when $r$ exists), and this became known as Eckhoff's conjecture. It was shown by Jamison [17] that the conjecture is true if $r=3$, and that the existence of $r$ always implies that $r_{k}$ exists and $r_{k} \leq r^{\left\lceil\log _{2} k\right\rceil} \leq(2 k)^{\log _{2} r}$. His proof used the recursion $r_{k l} \leq r_{k} r_{l}$ which was later improved by Eckhoff [13] to $r_{2 k+1} \leq(r-1)\left(r_{k+1}-1\right)+r_{k}+1$, but this did not significantly change the growth rate of the upper bound. Recently Bukh [7] disproved the conjectured bound $r_{k} \leq(r-1)(k-1)+1$ by showing an example where $r=4$, but $r_{k} \geq 3 k-1$ (just one more than the conjectured value), and also improved the upper bound to $r_{k}=O\left(k^{2} \log ^{2} k\right)$, where the hidden constant depends on $r$. We improve this to $r_{k}=O(k)$, which is optimal up to a constant factor and might lead to interesting applications.

Theorem 1.1 If a convexity space $(X, \mathcal{C})$ has Radon number $r$, then $r_{k} \leq c(r) \cdot k$.

Our proof combines the methods of Bukh with recent results of Holmsen and Lee [16]. In particular, we will use the following version of the classical fractional Helly theorem [18]. Here, and later, collection of $f$ sets $F_{1}, \ldots, F_{f} \in \mathcal{F}$ is an intersecting $f$-tuple from $\mathcal{F}$ if $\bigcap_{i=1}^{f} F_{i} \neq \emptyset$, i.e., they have a common point.

Theorem 1.2 (Holmsen-Lee [16]) For any $r \geq 3$ there is an $f$ such that for any $\alpha>0$ there is a $\beta>0$ with the following property. If a convexity space $(X, \mathcal{C})$ has Radon number $r$, then for any finite family $\mathcal{F}$ of convex sets, if at least an $\alpha$ fraction of the $f$-tuples of $\mathcal{F}$ are intersecting, then a $\beta$ fraction of $\mathcal{F}$ intersect in a common point.

There are several other connections between the parameters of a convexity space [29]. For example, the Helly number of a convexity space $(X, \mathcal{C})$ is defined as the smallest $h$ for which if all $h$-tuples of a finite family $\mathcal{F}$ of convex sets are intersecting, then all sets of $\mathcal{F}$ intersect in a common point. It was already shown in [21] that in all convexity spaces $h<r$ (if $r$ is finite), while in [16] it was also shown that the socalled colorful Helly number [4] can also be bounded by some function of $r$ (and this implied Theorem 1.2 combined with a combinatorial result from [15]). ${ }^{1}$ It was also shown in [16] that it follows from the work of Alon et al. [2] that weak $\varepsilon$-nets [1] of size $c(\varepsilon, r)$ also exist and a $(p, q)$-theorem [3] also holds, so understanding these parameters better might lead to improved $\varepsilon$-net bounds. It remains an interesting challenge and a popular topic to find new connections among such theorems; for some recent papers studying the Radon numbers or Tverberg theorems of various convexity spaces, see [9-11,14,20,23,24,27], while for a comprehensive survey, see Bárány and Soberón [6].

\subsection{Restricted vs. Multiset}

In case of general convexity spaces, there are two, slightly different definitions of Radon numbers $[29,5.19]$. When we do not allow repetitions in the point set $P$ to

\footnotetext{
${ }^{1}$ We would like to point out that a difficulty in proving these results is that the existence of a Carathéodorytype theorem is not implied by the existence of $r$.
} 
be partitioned, i.e., $P$ consist of different points, the parameter is called the restricted Radon number, which we will denote by $r_{k}^{(1)}$. If repetitions are also allowed, i.e., we want to partition a multiset, the parameter is called the unrestricted or multiset Radon number, which we will denote by $r_{k}^{(m)}$. The obvious connection between these parameters is $r_{k}^{(1)} \leq r_{k}^{(m)} \leq(k-1)\left(r_{k}^{(1)}-1\right)+1$. In the earlier papers multiset Radon numbers were preferred, while later papers usually focused on restricted Radon numbers; we followed the spirit of the age, so the results in the introduction were written using the definition of $r_{k}^{(1)}$, although some of the bounds (like Jamison's or Eckhoff's) are valid for both definitions. The proof of Theorem 1.1, however, also works for multisets, so we will in fact prove the stronger $r_{k}^{(m)}=O(k)$, and in the following simply use $r_{k}$ for the multiset Radon number $r_{k}^{(m)}$.

A similar issue arises in Theorem 1.2; is $\mathcal{F}$ allowed to be a multifamily? Though not emphasized in [16], their proof also works in this case and we will use it for a multifamily. Note that this could be avoided with some cumbersome tricks, like adding more points to the convexity space without increasing the Radon number $r$ to make all sets of a family different, but we do not go into details, as Theorem 1.2 holds for multifamilies anyway.

\section{Proof of Theorem 1.1}

Fix $r$, a convexity space $(X, \mathcal{C})$ with Radon number $r$, and a collection of points $P \subset X$ with cardinality $t k$, where we allow repetitions and the cardinality is understood as the sum of the multiplicities, so $P=\left\{p_{1}, \ldots, p_{t k}\right\}$. We will treat all points of $P$ as if they were different even if they coincide in $X$, e.g., when taking subsets.

We need to show that if $t \geq c(r)$, then we can partition $P$ into $k$ sets whose convex hulls intersect. For a fixed constant $s$, define $\mathcal{F}$ to be the family of convex sets that are the convex hulls of some $s$-element subset of $P$, i.e., $\mathcal{F}=\{\operatorname{conv} S|S \subset P| S \mid,=s\}$. Since we treat all points of $P$ as different, $\mathcal{F}$ will be a multifamily with $|\mathcal{F}|=\left(\begin{array}{c}t k \\ s\end{array}\right)$. We will refer to the point set $S$ whose convex hull gave some $F=\operatorname{conv} S \in \mathcal{F}$ as the vertices of $F$. Some of the points might be in the convex hull of the remaining ones; here vertices is meant in a graph theoretical sense. Note that for some $I, J \subset$ $\{1, \ldots, t k\}$ if we define $P_{I}=\left\{p_{i} \mid i \in I\right\}$ and $P_{J}=\left\{p_{j} \mid j \in J\right\}$, then we might have conv $P_{I}=$ conv $P_{J}$ even if $I \neq J$, but the vertices of conv $P_{I}$ and conv $P_{J}$ will still be $P_{I}$ and $P_{J}$; since $P$ is a multiset, this is even possible if $I \cap J=\emptyset$.

The constants $t$ and $s$ will be set to be large enough compared to some parameters that we get from Theorem 1.2 when we apply it to a fixed $\alpha$. (Our arguments work for any $0<\alpha<1$.) First we set $s$ to be large enough depending on $\alpha$ and $r_{f}$ (where $f$ is the fractional Helly number from Theorem 1.2; recall that $r_{f} \leq r^{\lceil\log f\rceil}$ is a constant [17]), then we set $t$ to be large enough depending on $s$ and $\beta$ (which depends on our chosen $\alpha$ ). In particular, we can set

$$
s=r_{f} f^{f r_{f}} \log \frac{1}{1-\alpha_{1}} \quad \text { and } \quad t=\max \left(\frac{s^{2}}{\beta} ; \frac{(f s)^{2}}{k\left(1-\alpha_{2}\right)}\right)
$$


where $0<\alpha_{1}, \alpha_{2}<1$ are any two numbers such that $\alpha_{1} \cdot \alpha_{2}=\alpha$. Also, we note that the proof from [15,16] gives $f \leq r^{r^{\log r}}$ and $\beta=\Omega\left(\alpha^{r^{f}}\right)$ for Theorem 1.2. Combining all these to get the best bound, note that $R=\max \left(r^{f}, f^{r_{f}}\right) \lesssim r^{r^{r^{\log r}}}$. Set $\alpha=1-1 / R$ with, e.g., $\alpha_{1}=\alpha_{2} \approx 1-1 /(2 R)$. This keeps $\beta$ constant, and both $s$ and $t$ around $R$,

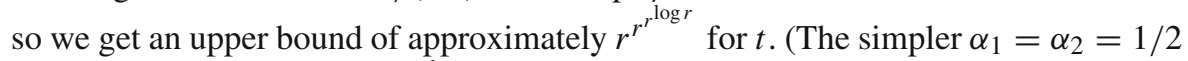
would give approximately $2^{r^{r^{\log r}}}$.)

Theorem 1.1 will be implied by the following lemma and Theorem 1.2. The proof is based on the observation that if a large point set is partitioned randomly into $k$ parts, then the convex hulls of the parts will intersect with high probability, because when we partition any $r_{k}$ points, there is a non-zero probability that their convex hulls will intersect. For related statements, see [5,19,25].

Lemma 2.1 An $\alpha$ fraction of the $f$-tuples of $\mathcal{F}$ are intersecting.

Proof Since $t$ is large enough, almost all $f$-tuples will be vertex-disjoint, thus it will be enough to deal with such $f$-tuples. More precisely, the probability of an $f$-tuple being vertex-disjoint is at least $(1-f s /(t k))^{f s} \geq 1-(f s)^{2} /(t k) \geq \alpha_{2}$ by the choice of $t$, using Bernoulli's inequality. We need to prove that at least an $\alpha_{1}$ fraction of these vertex-disjoint $f$-tuples will be intersecting.

Partition the vertex-disjoint $f$-tuples into groups depending on which $(f s)$-element subset of $P$ is the union of their vertices. We will show that for each group an $\alpha_{1}$ fraction of them are intersecting. We do this by generating the $f$-tuples of a group uniformly at random and show that such a random $f$-tuple will be intersecting with probability at least $\alpha_{1}$. For technical reasons, suppose that $m=s / r_{f}$ is an integer and partition the $f s$ supporting points of the group randomly into $m$ subsets of size $f r_{f}$, denoted by $V_{1}, \ldots, V_{m}$. Call an $f$-tuple type $\left(V_{1}, \ldots, V_{m}\right)$ if each set of the $f$-tuple intersects each $V_{i}$ in $r_{f}$ points. Since these $V_{i}$ were picked randomly, it is enough to show that the probability that a $\left(V_{1}, \ldots, V_{m}\right)$-type $f$-tuple is intersecting is at least $\alpha_{1}$.

The $\left(V_{1}, \ldots, V_{m}\right)$-type $f$-tuples can be uniformly generated by partitioning each $V_{i}$ into $f$ equal parts of size $r_{f}$, because sampling an $f$-tuple of vertex-disjoint sets uniformly at random is the same as first sampling a type uniformly at random and then sampling an $f$-tuple of that type uniformly at random. Therefore, it is enough to show that such a random $f$-tuple will be intersecting with probability at least $\alpha_{1}$. Since $\left|V_{i}\right| \geq r_{f}$, there is at least one partition of the first $r_{f}$ points of $V_{i}$ into $f$ parts whose convex hulls intersect. Since we can distribute the remaining $(f-1) r_{f}$ points of $V_{i}$ to make all $f$ parts equal, we get that when we partition $V_{i}$ into $f$ equal parts of size $r_{f}$, the convex hulls of these parts will intersect with probability at least $\left(\begin{array}{c}f r_{f} \\ r_{f}, r_{f}, \ldots, r_{f}\end{array}\right)^{-1} \geq f^{-f r_{f}}$, using that each of the $f r_{f}$ points can go to one of the $f$ parts. Since these events are independent for each $i$, we get that the final $f$-tuple will be intersecting with probability at least $1-\left(1-f^{-f r_{f}}\right)^{m} \geq 1-e^{-m f^{-f r_{f}}} \geq \alpha_{1}$ by the choice of $s$, using $1-x \geq e^{-x}$.

Therefore, if $s$ is large enough, the conditions of Theorem 1.2 are met, so at least $\beta\left(\begin{array}{c}t k \\ s\end{array}\right)$ members of $\mathcal{F}$ intersect in a common point. In other words, these intersecting sets form an $s$-uniform hypergraph $\mathcal{H}$ on $t k$ vertices that is $\beta$-dense. We need to show that 
$\mathcal{H}$ has $k$ disjoint edges to obtain the desired partition of $P$ into $k$ parts with intersecting convex hulls. For a contradiction, suppose that $\mathcal{H}$ has only $k-1$ disjoint edges. Then every other edge meets one of their $(k-1) s$ vertices. There are at most $(k-1) s\left(\begin{array}{c}t k \\ s-1\end{array}\right)$ such edges, which is less than $\beta\left(\begin{array}{c}t k \\ s\end{array}\right)$ if $(k-1) s<\beta(t k-s+1) / s$, but this holds by the choice of $t$. This finishes the proof of Theorem 1.1.

\section{Concluding Remarks}

It is an interesting question to study how big $f$ can be compared to $r$ and the Helly number $h$ of $(X, \mathcal{C})$. The current bound [16] gives $f \leq h^{r_{h}} \leq r^{r^{\log r}}$. We would like to point out that the first inequality, $f \leq h^{r_{h}}$, can be (almost) strict, as shown by the following example, similar to [22, Example 3] (cylinders). Let $X=\{1, \ldots, q\}^{d}$ be the points of a $d$-dimensional grid, and let $\mathcal{C}$ consist of the intersections of the axis-parallel affine subspaces with $X$. (Note that for $q=2, X$ will be the vertices of a $d$-dimensional cube, and $\mathcal{C}$ its faces.) It is easy to check that $h=2, r=\lfloor\log (d+1)+2\rfloor$, and $f=d+1$ : we have $h=2$ because a family $\mathcal{F}$ is (pairwise) intersecting if and only if there are no $F_{1}, F_{2} \in \mathcal{F}$ that are contained in different parallel hyperplanes $H_{1} \supset F_{1}$ and $H_{2} \supset F_{2}$; we have $r=\lfloor\log (d+1)+2\rfloor$ because the number of ways $r$ points can be partitioned into two non-empty parts is $2^{r-1}-1>d$, so in some partition no coordinate will be a different constant on the two parts, thus the convex hulls will intersect; we have $f=d+1$ because in Theorem 1.2 for $\alpha=d ! / d^{d}$ we need $\beta=1 / q$ when $\mathcal{F}$ consists of all $q d$ axis-parallel affine hyperplanes (if $q$ is large enough).

It is tempting to assume that Theorem 1.1 would improve the second inequality, $h^{r_{h}} \leq r^{r^{\log r}}$, as instead of $r_{h} \leq r^{\log h}$ we can use $r_{h}=O(h)$. Unfortunately, recall that the hidden constant depended on $r$, in particular, it is around $r^{r^{\log r}}$. We have a suspicion that this might not be entirely sharp, so a natural question is whether this dependence could be removed to improve $r_{k} \leq r^{r^{\log r}} \cdot k$ to $r_{k} \leq c \cdot r \cdot k$. This would truly lead to an improvement of the upper bound on $f$ in Theorem 1.2 and would lead to further applications [6].

Acknowledgements I would like to thank Boris Bukh and Narmada Varadarajan for discussions on [7], Andreas Holmsen for calling my attention to the difference between restricted and multiset Radon numbers, especially for confirming that Theorem 1.2 also holds for multisets, Imre Bárány for calling my attention to [5,19,25], and Gábor Damásdi, Balázs Keszegh, Padmini Mukkamala, and Géza Tóth for feedback on earlier versions of this manuscript, especially for fixing the computations in the proof of Lemma 2.1. I would also like to thank my anonymous referees for several valuable suggestions that improved the presentation of the paper.

Funding Open access funding provided by the Eötvös Loránd University.

Open Access This article is licensed under a Creative Commons Attribution 4.0 International License, which permits use, sharing, adaptation, distribution and reproduction in any medium or format, as long as you give appropriate credit to the original author(s) and the source, provide a link to the Creative Commons licence, and indicate if changes were made. The images or other third party material in this article are included in the article's Creative Commons licence, unless indicated otherwise in a credit line to the material. If material is not included in the article's Creative Commons licence and your intended use is not permitted 
by statutory regulation or exceeds the permitted use, you will need to obtain permission directly from the copyright holder. To view a copy of this licence, visit http://creativecommons.org/licenses/by/4.0/.

\section{References}

1. Alon, N., Bárány, I., Füredi, Z., Kleitman, D.J.: Point selections and weak $\epsilon$-nets for convex hulls. Comb. Probab. Comput. 1(3), 189-200 (1992)

2. Alon, N., Kalai, G., Matoušek, J., Meshulam, R.: Transversal numbers for hypergraphs arising in geometry. Adv. Appl. Math. 29(1), 79-101 (2002)

3. Alon, N., Kleitman, D.J.: Piercing convex sets and the Hadwiger-Debrunner $(p, q)$-problem. Adv. Math. 96(1), 103-112 (1992)

4. Bárány, I.: A generalization of Carathéodory’s theorem. Discrete Math. 40(2-3), 141-152 (1982)

5. Bárány, I.: Tensors, colours, octahedra. In: Geometry, Structure and Randomness in Combinatorics (Pisa 2012). CRM Series, vol. 18, pp. 1-17. Edizioni della Normale, Pisa (2015)

6. Bárány, I., Soberón, P.: Tverberg's theorem is 50 years old: a survey. Bull. Am. Math. Soc. 55(4), 459-492 (2018)

7. Bukh, B.: Radon partitions in convexity spaces (2010). arXiv:1009.2384

8. Calder, J.R.: Some elementary properties of interval convexities. J. Lond. Math. Soc. 3, 422-428 (1971)

9. De Loera, J.A., La Haye, R.N., Rolnick, D., Soberón, P.: Quantitative combinatorial geometry for continuous parameters. Discrete Comput. Geom. 57(2), 318-334 (2017)

10. De Loera, J.A., La Haye, R.N., Rolnick, D., Soberón, P.: Quantitative Tverberg theorems over lattices and other discrete sets. Discrete Comput. Geom. 58(2), 435-448 (2017)

11. De Loera, J.A., Hogan, T.A., Meunier, F.A., Mustafa, N.H.: Tverberg theorems over discrete sets of points. In: Polytopes and Discrete Geometry. Contemporary Mathematics, vol. 764, pp. 57-70. American Mathematical Society, Providence (2021)

12. Eckhoff, J.: Radon's theorem revisited. In: Contributions to Geometry (Siegen 1978), pp. 164-185. Birkhäuser, Basel (1979)

13. Eckhoff, J.: The partition conjecture. Discrete Math. 221(1-3), 61-78 (2000)

14. Fulek, R., Gärtner, B., Kupavskii, A., Valtr, P., Wagner, U.: The crossing Tverberg theorem. In: 35 th International Symposium on Computational Geometry (Portland 2019). Leibniz International Proceedings in Informatics, vol. 129, \# 38. Leibniz-Zent. Inform., Wadern (2019)

15. Holmsen, A.F.: Large cliques in hypergraphs with forbidden substructures. Combinatorica 40(4), 527537 (2020)

16. Holmsen, A.F., Lee, D.: Radon numbers and the fractional Helly theorem. Isr. J. Math. 241(1), 433-447 (2021)

17. Jamison-Waldner, R.E.: Partition numbers for trees and ordered sets. Pac. J. Math. 96(1), 115-140 (1981)

18. Katchalski, M., Liu, A.: A problem of geometry in $R^{n}$. Proc. Am. Math. Soc. 75(2), 284-288 (1979)

19. Kirchberger, P.: Über Tchebychefsche Annäherungsmethoden. Math. Ann. 57(4), 509-540 (1903)

20. Letzter, S.: Radon numbers for trees. Discrete Math. 340(3), 333-344 (2017)

21. Levi, F.W.: On Helly's theorem and the axioms of convexity. J. Indian Math. Soc. 15, 65-76 (1951)

22. Moran, S., Yehudayoff, A.: On weak $\epsilon$-nets and the Radon number. In: 35 th International Symposium on Computational Geometry (Portland 2019). Leibniz International Proceedings in Informatics, vol. 129, \# 51. Leibniz-Zent. Inform., Wadern (2019)

23. Paták, P.: Properties of closure operators in the plane (2019). arXiv:1909.08489

24. Patáková, Z.: Bounding Radon number via Betti numbers. In: 36th International Symposium on Computational Geometry. Leibniz International Proceedings in Informatics, vol. 164, \# 61. Leibniz-Zent. Inform., Wadern (2020)

25. Pór, A.: Diploma thesis. Eötvös Loránd University, Budapest (1998)

26. Radon, J.: Mengen konvexer Körper, die einen gemeinsamen Punkt enthalten. Math. Ann. 83(1-2), 113-115 (1921)

27. Soberón, P.: Tverberg partitions as weak epsilon-nets. Combinatorica 39(2), 447-458 (2019)

28. Tverberg, H.: A generalization of Radon's theorem. J. Lond. Math. Soc. 41, 123-128 (1966) 
29. van de Vel, M.L.J.: Theory of Convex Structures. North-Holland Mathematical Library, vol. 50. NorthHolland, Amsterdam (1993)

Publisher's Note Springer Nature remains neutral with regard to jurisdictional claims in published maps and institutional affiliations. 